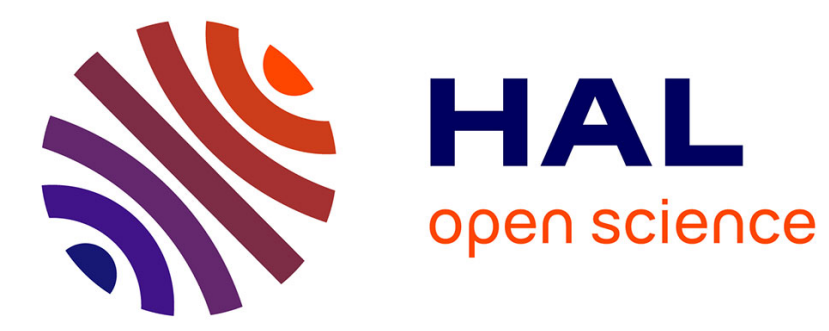

\title{
Acoustoelasticity in transverse isotropic soft tissues: quantification of muscles' nonlinear elasticity
}

Marion Bied, Laurène Jourdain, Jean-Luc Gennisson

\section{To cite this version:}

Marion Bied, Laurène Jourdain, Jean-Luc Gennisson. Acoustoelasticity in transverse isotropic soft tissues: quantification of muscles' nonlinear elasticity. 2020 IEEE International Ultrasonics Symposium (IUS), Sep 2020, Las Vegas (virtual), United States. pp.1-4, 10.1109/IUS46767.2020.9251574 . hal-03096307

\section{HAL Id: hal-03096307 https://hal.science/hal-03096307}

Submitted on 4 Jan 2021

HAL is a multi-disciplinary open access archive for the deposit and dissemination of scientific research documents, whether they are published or not. The documents may come from teaching and research institutions in France or abroad, or from public or private research centers.
L'archive ouverte pluridisciplinaire HAL, est destinée au dépôt et à la diffusion de documents scientifiques de niveau recherche, publiés ou non, émanant des établissements d'enseignement et de recherche français ou étrangers, des laboratoires publics ou privés. 


\title{
Acoustoelasticity in transverse isotropic soft tissues: quantification of muscles' nonlinear elasticity
}

\author{
Marion Bied, Laurène Jourdain, Jean-Luc Gennisson \\ Laboratoire d'Imagerie Biomédicale Multimodale, BioMaps, Université Paris-Saclay, CEA, \\ CNRS UMR 9011, Inserm UMR1281, SHFJ, 4 place du général Leclerc, 91401, Orsay, France \\ jean-luc.gennisson@universite-paris-saclay.fr Orcid : 0000-0001-8318-8237
}

\begin{abstract}
Quantification of the elastic nonlinearity of biological tissues is a recent refinement of measures available in ultrasound elastography. The measurement of the third order nonlinear elastic modulus of biological tissues using ultrasound elastography relies on acoustoelasticity (AE), consisting in measuring the shear wave velocity in tissues under uniaxial stress. Up to now, the AE theory has been developed under the hypothesis of tissue isotropy. However, this assumption does not hold for all tissues, such as muscles. Indeed, the direction of alignment of muscular fibers defines a rotation symmetry axis, justifying the modelling of muscles in terms of transverse isotropic (TI) media. Such tissues require a more complex elastic description, considering their anisotropy. In this work, the $\mathrm{AE}$ theory is transposed to quasi-incompressible TI media and tested experimentally. After developing the elastic energy of TI quasiincompressible media up to the third order, the relations between the shear wave speed and the applied stress are derived. In addition to the 3 linear elastic moduli describing a quasiincompressible TI medium, 4 nonlinear elastic moduli appear. This theoretical development is tested experimentally on TI PVA phantoms and on $e x$ vivo beef muscle tissues using an experimental setup designed to apply controlled uniaxial stresses to the sample, and one of the third order nonlinear coefficients, coefficient $A$, is estimated. This work contributes to opening the path towards the nonlinear characterization of muscles, which has potential applications in muscle biomechanics and in muscular pathology diagnosis.
\end{abstract}

Keywords-acoustoelasticity, elastic nonlinearity, muscle, transverse isotropy, ultrasound elastography, ultrafast imaging

\section{INTRODUCTION}

Quantification of the elastic nonlinearity of biological tissues is of increasing interest in the early diagnosis of pathologies, such as breast lesions [1]. Measurement of nonlinear shear modulus (NLSM) in biological tissues using shear wave elastography relies on acoustoelasticity (AE). It consists in measuring the shear wave velocity $v_{s}$ under uniaxial stress. The $\mathrm{AE}$ theory has been previously developed in isotropic quasiincompressible materials [2]. In the development, 3 configurations differing in the relative orientation of the uniaxial stress with respect to the polarization and the propagation directions of the shear wave have been identified, and the corresponding relationships between $v_{s}$, the local stress $(\sigma)$, the linear shear modulus $(\mu)$ and the $\operatorname{NLSM}(A)$ has been derived for each case. In transverse isotropic (TI) medium, such as muscle, this approach is no more valid due to anisotropy. Indeed, in muscles, the alignment of fibers defines a rotation symmetry axis. The description of muscles in terms of elasticity is therefore more complex, but has always to be in line with their geometry. The goal of this work is to transpose the AE theory to TI soft tissues for muscle elastic nonlinearity quantification. This implies considering the constraints imposed by the TI geometry of muscles in the AE development.

\section{THEORETICAL BACKGROUND}

The AE theory derived here for TI quasi-incompressible media is highly inspired from Gennisson et al. development in isotropic media [2]. The development aims at expressing the speed of elastic shear waves in an uniaxially stressed TI quasiincompressible solid assumed to be lossless. In the following section, a coordinate system which third axis coincides with the symmetry axis of the considered TI medium is used (Fig. 1).

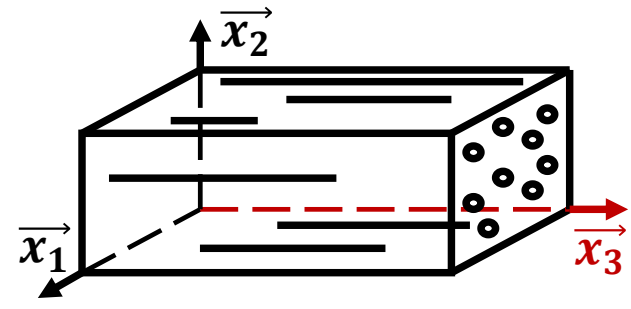

Fig. 1. Coordinate system employed in part II for a TI medium.

The theoretical development of $\mathrm{AE}$ is based on the expression of the volume strain energy $(e)$ of the studied medium developed up to the third order in terms of strain (1). By considering the symmetries imposed by the anisotropy of the TI medium and the constraints due to incompressibility, the strain energy of a TI incompressible medium is expressed as (adapted from [3]):

$$
\begin{gathered}
e=\mu_{\perp} I_{2}+\left(\frac{E_{\|}}{2}-\frac{3 \mu_{\perp}}{2}\right) \varepsilon_{33}^{2}+2\left(\mu_{\|}-\mu_{\perp}\right)\left(\varepsilon_{13}^{2}+\varepsilon_{23}^{2}\right) \\
+\frac{A}{3} I_{3}+(G+H+J) \varepsilon_{33}^{3}+(H+2 J) \varepsilon_{33}\left(\varepsilon_{13}^{2}+\varepsilon_{23}^{2}\right) \\
+J \varepsilon_{33}\left(\varepsilon_{11}^{2}+\varepsilon_{22}^{2}+2 \varepsilon_{12}^{2}\right)
\end{gathered}
$$


where $\varepsilon$ is the Green-Lagrange strain tensor, $I_{2}=\operatorname{Tr} \varepsilon^{2}$ and $I_{3}=\operatorname{Tr} \varepsilon^{3}, 2$ of the 3 invariants of $\varepsilon$ defined by Landau and Lifshitz [4]. $E_{/ /}$(Young's modulus parallel to the principal axis), $\mu_{\perp}$ and $\mu_{/ /}$(shear moduli perpendicular and parallel to the principal axis, respectively) are the 3 linear elastic independent coefficients sufficient to fully describe the linear elastic behavior of a TI incompressible medium. $A, G, H$ and $J$ are 4 third order (nonlinear) elastic coefficients.

From the expression of $e$, the first Piola-Kirchhoff stress tensor $\left(P_{i k}\right)$ is derived (2):

$$
P_{i k}=\frac{\partial e}{\partial\left(\frac{\partial u_{i}}{\partial a_{k}}\right)}
$$

Where $(\vec{u})$ is the displacement vector and $(\vec{a})$ the position in Lagrangian coordinates. The expression of $P_{i k}$ is then employed to obtain the motion equation of particles in Lagrangian coordinates using the expression (Einstein's summation convention of repeated indices is used):

$$
\rho_{0} \ddot{u}_{\imath}=\frac{\partial P_{i k}}{\partial a_{k}}
$$

where $\left(\rho_{0}\right)$ is the medium's density and (ii) the acceleration vector.

After distinguishing the dynamical (due to the propagating shear wave) and static (due to uniaxial stress) components of the displacement $\vec{u}$, a change of coordinates from Lagrangian to Eulerian coordinates yields the wave equation from which can be deduced directly the shear wave velocity. At this stage, static strain terms (due to uniaxial stress) appear in the equations. The latter can be expressed in terms of the applied stress $(\sigma)$ by applying Hooke's law in TI quasi-incompressible

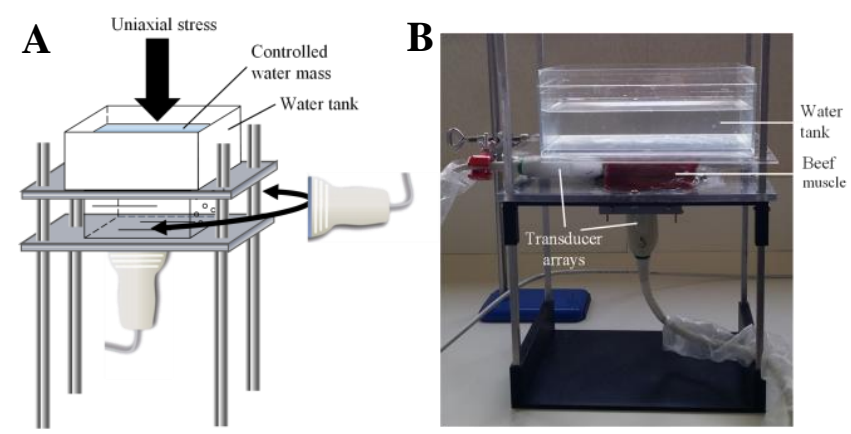

Fig. 2. Experimental setup used to generate controlled uniaxial stresses on the investigated TI media and to measure the shear wave speed. (A) Diagram of the setup. (B) Picture of the setup.

media. Rather than expressing shear wave speed in all cases (which would require projections and complex expressions), we have concentrated ourselves on 9 specific configurations differing in the relative orientations of stress $(\sigma)$, polarization $(\vec{u})$ and propagation $(\vec{k})$ of the shear wave with respect to the principal axis of the TI medium (table 1). The mentioned reasoning yields the 9 elastodynamic equations specified in table 1 .

As for isotropic media [2], the apparent shear modulus $\rho_{o} v_{s}{ }^{2}$ depends linearly on the applied uniaxial stress in TI media. The stress $\sigma$ multiplying coefficients depend on 3 nonlinear elastic coefficients, as well as the 3 linear elastic moduli of a TI medium. By having access to all configurations and knowing $\mu_{/ /, \mu}$, and $E_{/ /}$these relations open the path to a full nonlinearity quantification of TI soft tissues.

\section{MATERIAL AND METHODS}

To validate the AE theory derived above and make use of it, AE experiments were carried out on TI quasi-incompressible media. Such AE experiments require a set-up allowing to apply

\begin{tabular}{|c|c|c|c|c|}
\hline \multicolumn{4}{|c|}{ Configuration } & \multirow{3}{*}{ Elastodynamic equations } \\
\hline \multicolumn{3}{|c|}{ Direction } & \multirow{2}{*}{$\#$} & \\
\hline $\overrightarrow{\boldsymbol{u}}$ & $\overrightarrow{\boldsymbol{k}}$ & $\sigma$ & & \\
\hline \multirow{3}{*}{$\overrightarrow{x_{2}}$} & \multirow{3}{*}{$\overrightarrow{x_{3}}$} & $\overrightarrow{x_{1}}$ & 1 & $\rho_{0} v_{s}^{2}=\mu_{\|}+\frac{\sigma_{11}}{2 E_{\|}}\left(E_{\|}-\mu_{\perp}+\mu_{\|}+\frac{\mu_{\|} E_{\|}}{\mu_{\perp}}+\frac{A}{4}\left(1+\frac{E_{\|}}{\mu_{\perp}}\right)+\frac{H+2 J}{2}\right)$ \\
\hline & & $\overrightarrow{x_{2}}$ & 2 & $\rho_{0} v_{s}^{2}=\mu_{\|}+\frac{\sigma_{22}}{2 E_{\|}}\left(E_{\|}-\mu_{\perp}+\mu_{\|}-\frac{\mu_{\|} E_{\|}}{\mu_{\perp}}+\frac{A}{4}\left(1-\frac{E_{\|}}{\mu_{\perp}}\right)+\frac{H+2 J}{2}\right)$ \\
\hline & & $\overrightarrow{x_{3}}$ & 3 & $\rho_{0} v_{s}^{2}=\mu_{\|}-\frac{\sigma_{33}}{E_{\|}}\left(E_{\|}-\mu_{\perp}+\mu_{\|}+\frac{A}{4}+\frac{H+2 J}{2}\right)$ \\
\hline \multirow{3}{*}{$\overrightarrow{x_{3}}$} & \multirow{3}{*}{$\overrightarrow{x_{2}}$} & $\overrightarrow{x_{1}}$ & 4 & $\rho_{0} v_{s}^{2}=\mu_{\|}+\frac{\sigma_{11}}{2 E_{\|}}\left(E_{\|}-\mu_{\perp}+\mu_{\|}+\frac{\mu_{\|} E_{\|}}{\mu_{\perp}}+\frac{A}{4}\left(1+\frac{E_{\|}}{\mu_{\perp}}\right)+\frac{H+2 J}{2}\right)$ \\
\hline & & $\overrightarrow{x_{2}}$ & 5 & $\rho_{0} v_{s}^{2}=\mu_{\|}+\frac{\sigma_{22}}{2 E_{\|}}\left(-E_{\|}-\mu_{\perp}+\mu_{\|}-\frac{\mu_{\|} E_{\|}}{\mu_{\perp}}+\frac{A}{4}\left(1-\frac{E_{\|}}{\mu_{\perp}}\right)+\frac{H+2 J}{2}\right)$ \\
\hline & & $\overrightarrow{x_{3}}$ & 6 & $\rho_{0} v_{s}^{2}=\mu_{\|}-\frac{\sigma_{33}}{E_{\|}}\left(-\mu_{\perp}+\mu_{\|}+\frac{A}{4}+\frac{H+2 J}{2}\right)$ \\
\hline \multirow{3}{*}{$\overrightarrow{x_{2}}$} & \multirow{3}{*}{$\overrightarrow{x_{1}}$} & $\overrightarrow{x_{1}}$ & 7 & $\rho_{0} v_{s}^{2}=\mu_{\perp}-\frac{\sigma_{11}}{2 E_{\|}}\left(E_{\|}+3 \mu_{\perp}+\frac{A}{2}-J\right)$ \\
\hline & & $\overrightarrow{x_{2}}$ & 8 & $\rho_{0} v_{s}^{2}=\mu_{\perp}-\frac{\sigma_{22}}{2 E_{\|}}\left(-E_{\|}+3 \mu_{\perp}+\frac{A}{2}-J\right)$ \\
\hline & & $\overrightarrow{x_{3}}$ & 9 & $\rho_{0} v_{s}^{2}=\mu_{\perp}+\frac{\sigma_{33}}{E_{\|}}\left(3 \mu_{\perp}+\frac{A}{2}-J\right)$ \\
\hline
\end{tabular}

TABLE I. Elastodynamic EQUATIONS For THE 9 DEFINED CONFIGURATIONS 
known uniaxial stress on the studied medium and to measure the shear wave speed in the stresses medium.

\section{A. Studied TI media}

AE experiments were carried out on TI PVA phantoms and on fresh bovine ex vivo muscular tissues.

TI PVA phantoms were produced following [5]. PVA (Polyvinyl alcohol hydrolyzed, Sigma-Aldrich, St Louis, MO) (10\% in mass) is dissolved in heated water $\left(95^{\circ} \mathrm{C}\right)$ and Sigmacell (type 20) (Sigmacell Cellulose type 20, SigmaAldrich, St Louis, MO) (1\% in mass) is added as scatterers in the cooled solution (at room temperature). PVA phantoms underwent 3 isotropic freezing-thawing cycles (15 hours at $18^{\circ} \mathrm{C}, 9$ hours at $25^{\circ} \mathrm{C}$ ) followed by 3 anisotropic ones. During the latter, phantoms are placed in a jaw system and stretched in one direction to the maximum (Fig. 3A). This causes PVA to polymerize in the stretching direction.

\section{B. Applying known uniaxial stress in all 9 configurations}

An experimental set-up inspired from [2] was conceived and built (Fig. 2). Briefly, the studied sample lies on a plexiglass plate. To generate known uniaxial (vertical) stress, a second plexiglass plate is placed above the sample. A water tank covering the entire sample surface $S$ is added above this plate and filled with a controlled water mass. The applied stress can be computed: $\sigma=\frac{g m}{s}$, with $g$ the gravity of Earth, $m$ the whole mass above the sample.

SL10-2 ultrasound probes (6 MHz central frequency, 192 elements) (SuperSonic Imagine, Aix-en-Provence, France), are placed either below the sample or on the side and oriented as desired with respect to the principal axis of the studied medium that is detected beforehand by eye (manually). As mentioned below, shear wave speed is measured using the Supersonic Imaging (SSI) technique: therefore, the orientation of the probe defines the polarization direction (probe axis) and the considered propagation direction (lateral direction) of the generated shear wave. Measurements can be carried out in any of the 9 defined configurations, provided the system is stable and the sample's dimensions are large enough.

A

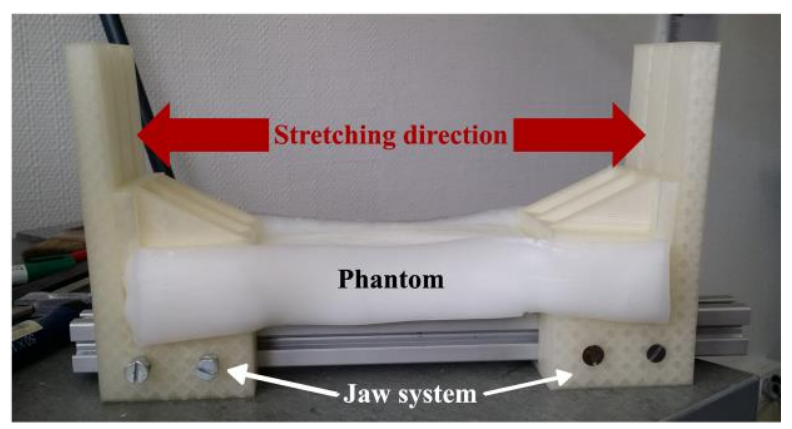

TABLE II. LINEAR $\left(\mu / /, \mu_{\perp}\right)$ AND NONLINEAR $(A)$ ELASTIC MODULI ESTIMATED IN THE INVESTIGATED TI MEDIA

\begin{tabular}{|c|l|c|c|}
\hline & TI PVA Phantom & Beef muscle 1 & Beef muscle 2 \\
\hline $\boldsymbol{\mu}_{/ /}(\mathbf{k P a})$ & $21.4 \pm 0.3(1,2)^{\mathrm{a}}$ & $60.0 \pm 19.0(1,2)^{\mathrm{a}}$ & $65.5 \pm 0.4(2)^{\mathrm{a}}$ \\
\hline $\boldsymbol{\mu}_{\perp}(\mathbf{k P a})$ & $14.2 \pm 0.1(8)^{\mathrm{a}}$ & $22.1 \pm 5.0(8,9)^{\mathrm{a}}$ & $24.5 \pm 0.1(8,9)^{\mathrm{a}}$ \\
\hline $\mathbf{A}(\mathbf{k P a})$ & $-33.0 \pm 17.1(1,2)^{\mathrm{a}}$ & $-2.8 .10^{3} \pm$ & $-1.6 .10^{3} \pm$ \\
& & $2.5 .10^{3}(1,2)^{\mathrm{a}}$ & $0.6 .10^{3}(1,2)^{\mathrm{a}}$ \\
\hline
\end{tabular}

\section{Measuring the shear wave speed}

The shear wave speed in stresses media is measured using the Supersonic Shear Imaging technique [6] available on the ultrafast ultrasound scanning device Aixplorer V12 (SuperSonic Imagine, Aix-en-Provence, France). The focusing of ultrasound at 4 successive depths creates an ultrasonic radiation force, generating a quasi-cylindrical shear wave which polarization is aligned with the probe axis. The latter is imaged at a high frame rate $(8 \mathrm{kHz})$ in the imaging plane using ultrafast ultrasound imaging. Thanks to a time of flight algorithm, the local shear wave speed is retrieved, and shear wave speed maps are obtained. For each AE measurement series (for one sample, in one configuration for different stress), a region of interest (ROI) is defined, and the mean shear wave speed is computed. The apparent shear modulus $\rho_{0} v_{s}^{2}$ is then deduced.

\section{Acoustoelasticity analysis}

The relations between the measured apparent shear moduli and the applied stress are analyzed based on the previously developed AE theory in TI quasi-incompressible media. In each configuration and for each sample, a least-squares linear regression is used to fit experimental data and to retrieve the slopes. The latter are used for quantification of the nonlinear elastic coefficient $A$.

\section{RESULTS}

Representative results of $\mathrm{AE}$ experiments in TI media are presented in figure 3B for TI PVA phantoms and in figure 4 for bovine muscular tissues. Shear moduli measured in no stress conditions allow to characterize the studied medium's anisotropy $\left(\mu_{/ /}\right.$over $\mu_{\perp}$ ratio of approximatively 1.5 for PVA

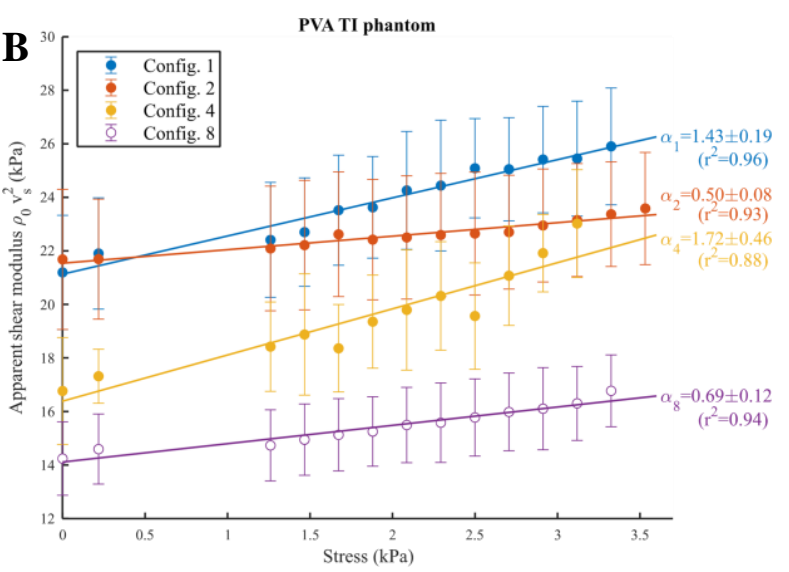

Fig. 3. 2 AE experiments on TI PVA phantoms. (A) Experimental setup used to stretch the PVA phantom during the 3 anisotropic freezing-thawing cycles. (B) Evolution of the apparent shear modulus of a TI PVA phantom as a function of the applied stress for each measured configuration. The mean apparent shear modulus of the considered ROI is represented. Error bars indicate the standard deviation over the ROI. Slopes obtained from the least-squares linear regression \pm the $95 \%$ confidence interval are specified, along with the determination coefficient $r^{2}$. 

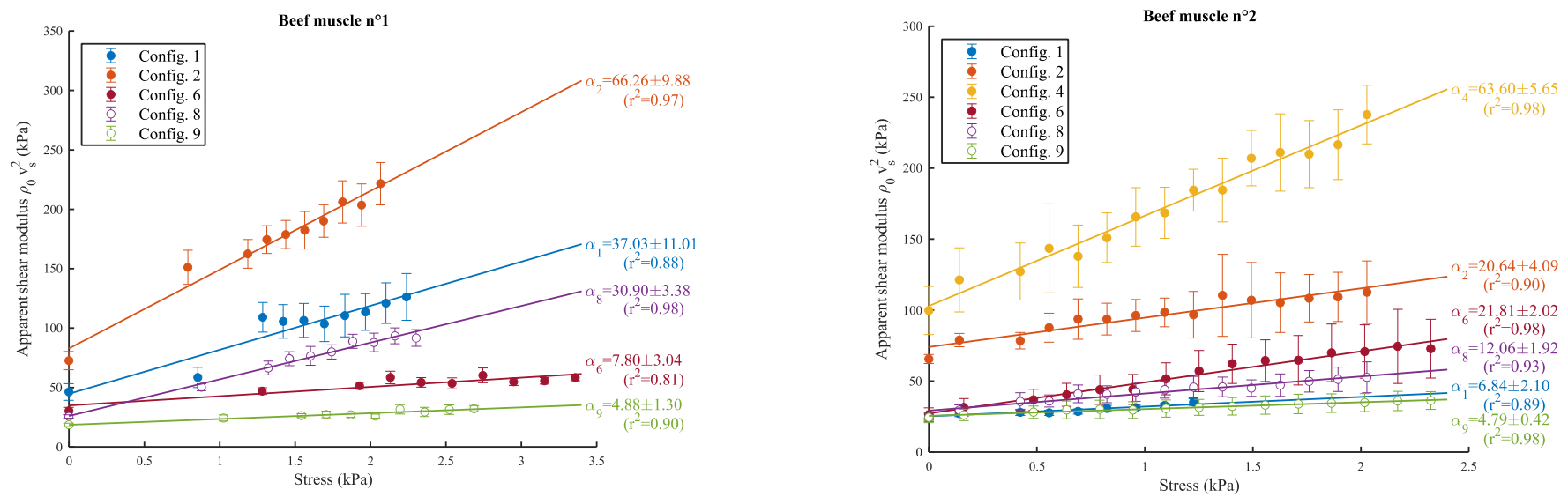

Fig. 4. AE experiment results on 2 ex vivo bovine muscular tissues. Measured apparent shear modulus of 2 bovine muscular tissues as a function of the applied stress for each measured configuration. The mean apparent shear modulus on the considered ROI is represented. Error bars indicate the corresponding standard deviation. Slopes obtained from the least-squares linear regression \pm the $95 \%$ confidence interval are specified, along with the determination coefficient $r^{2}$.

phantoms, and of 3 for bovine muscles). Globally, a linear relationship between the apparent shear modulus $\rho_{o} v_{s}^{2}$ and the applied uniaxial stress $\sigma$ is found in all investigated configurations (determination coefficients $r^{2}$ above 0.8 ), as predicted in the theory. The estimated slopes of configurations 1 and 2 are used to quantify the third order nonlinear coefficient $A$ of the TI medium (table 2).

\section{DISCUSSION}

The AE theory in TI quasi-incompressible medium was successfully derived, leading to the expression of the shear wave speed as a function of stress in 9 specific configurations. In addition to the 3 independent linear elastic moduli $\left(\mu_{/ /,} \mu_{\perp}, E_{/ /}\right)$ describing the linear elastic behavior of a quasi-incompressible TI medium, 3 third order (nonlinear) elastic coefficients appear in the outcoming equations, opening the path to TI tissue elastic nonlinearity quantification. AE experiments were carried out on TI PVA phantoms and bovine muscular tissues. As expected from the theory, a linear relationship between the apparent shear modulus and the stress was found, and the slopes of the experimental curves were used to retrieve the nonlinear elastic coefficient $A$ of the TI studied media. The obtained values in beef muscle are globally one order of magnitude higher (in terms of absolute value) compared to ex vivo measures of $A$ in beef isotropic tissues (liver) [7]. To our knowledge, these are the first nonlinear elasticity measurements carried out in TI tissues.

However, the quality of our estimations of $A$ relies on the precision with which the stress and the probe (fixing the considered directions of propagation and polarization of shear waves) are oriented with respect to the symmetry axis of the TI medium. Indeed, any deviation of the experimental positions from the theoretical configurations results in the estimated slopes being a combination of the expressions related to 2 or more configurations, leading to errors in $A$ estimation. The combination of Backscatter Tensor Imaging (BTI) [7] or Elastic Tensor Imaging (ETI) [8] with AE experiments in TI tissues would help to better match the experimental positions with the theoretical configurations and therefore improve the precision of $A$ estimation. (Note that the use of configuration 6 along with configurations 1 or 2 leads to different estimations of $A$. The difficulties to match experimentally configuration 6 can account for the differences observed in the estimations of $A$.) Besides, to fully take advantage of the $\mathrm{AE}$ theory and recover $H$ and $J$, the independent estimation of $E_{/ /}$is necessary. However, such a measurement is challenging since it requires lateral strain estimation. This can be carried out by using static elastography but remains delicate because of the poor ultrasound lateral resolution.

Nevertheless, this work paves the way to use the AE theory for a better and more complete muscle characterization in biomechanics, clinics and sport applications.

\section{ACKNOWLEDGEMENTS}

Authors would like to sincerely thanks X. Jacob, B. Giammarinaro, S. Chatelin and S. Catheline for useful discussions and interactions.

\section{REFERENCES}

[1] M. Bernal et al., "In Vivo Quantification of the Nonlinear Shear Modulus in Breast Lesions: Feasibility Study," IEEE Trans. Ultrason. Ferroelectr. Freq. Control, vol. 63, no. 1, pp. 101-109, Jan. 2016, doi: 10.1109/TUFFC.2015.2503601.

[2] J.-L. Gennisson et al., "Acoustoelasticity in soft solids: Assessment of the nonlinear shear modulus with the acoustic radiation force," J. Acoust. Soc. Am., vol. 122, pp. 3211-9, Jan. 2008, doi: 10.1121/1.2793605.

[3] G. C. Johnson, "Acoustoelastic response of polycrystalline aggregates exhibiting transverse isotropy," J. Nondestruct. Eval., vol. 3, no. 1, pp. 18, Mar. 1982, doi: 10.1007/BF00566949.

[4] L D Landau and E.M. Lifshitz, Theory of Elasticity 3rd Edition. Butterworth-Heinemann, 2012.

[5] S. Chatelin et al., "Anisotropic polyvinyl alcohol hydrogel phantom for shear wave elastography in fibrous biological soft tissue: a multimodality characterization," Phys. Med. Biol., vol. 59, no. 22, pp. 6923-6940, Oct. 2014, doi: 10.1088/0031-9155/59/22/6923.

[6] J. Bercoff et al., "Supersonic shear imaging: a new technique for soft tissue elasticity mapping," IEEE Trans. Ultrason. Ferroelectr. Freq. Control, vol. 51, no. 4, pp. 396-409, Apr. 2004, doi: 10.1109/tuffc.2004.1295425.

[7] C. Papadacci et al., "Ultrasound backscatter tensor imaging (BTI): analysis of the spatial coherence of ultrasonic speckle in anisotropic soft tissues," IEEE Trans. Ultrason. Ferroelectr. Freq. Control, vol. 61, no. 6, pp. 986-996, Jun. 2014, doi: 10.1109/TUFFC.2014.2994.

[8] M. Correia et al., "3D elastic tensor imaging in weakly transversely isotropic soft tissues," Phys. Med. Biol., vol. 63, no. 15, p. 155005, Jul. 2018, doi: 10.1088/1361-6560/aacfaf. 